Public Health Ethics. Scientific methods, foundational concepts, and case analyses. An interdisciplinary European conference for young scholars. Hannover, 14.-18. Februar 2011

Wild, V

DOI: https://doi.org/10.1007/s00481-011-0151-y

Posted at the Zurich Open Repository and Archive, University of Zurich ZORA URL: https://doi.org/10.5167/uzh-51362

Journal Article

Published Version

Originally published at:

Wild, V (2011). Public Health Ethics. Scientific methods, foundational concepts, and case analyses. An interdisciplinary European conference for young scholars. Hannover, 14.-18. Februar 2011. Ethik in der Medizin, 23(4):333-335.

DOI: https://doi.org/10.1007/s00481-011-0151-y 


\section{Public Health Ethics. Scientific methods, foundational concepts, and case analyses. An interdisciplinary European conference for young scholars}

\section{Hannover, 14.-18. Februar 2011}

\section{Verina Wild}

Online publiziert: 24. September 2011

(C) Springer-Verlag 2011

Vom 14. bis 18. Februar 2011 fand an der Medizinischen Hochschule Hannover die durch das Bundesministerium für Bildung und Forschung geförderte Klausurtagung „Public Health Ethics. Scientific methods, foundational concepts, and case analyses“ statt. Die Konferenz für 14 internationale Nachwuchswissenschaftlerinnen und -wissenschaftler wurde von Daniel Strech, Irene Hirschberg (beide Medizinische Hochschule Hannover) und Georg Marckmann (Ludwig-Maximilians-Universität München) organisiert.

Die thematische Abgrenzung des Forschungsfeldes Public Health Ethik, die zugrunde liegenden Konzepte und Theorien und die wissenschaftlichen Methoden sind Gegenstand eines international zunehmenden Diskurses [1, 3-5]. In Deutschland hat die Auseinandersetzung mit Public Health Ethik Fragen erst in den letzten Jahren begonnen [2, 7]. Die Tagung traf damit den Nerv der Zeit und hat nicht nur den Teilnehmenden einen beachtlichen Wissenszuwachs ermöglicht, sondern sie war auch dem entstehenden Feld der Public Health Ethik förderlich, u. a. dadurch, dass neu geknüpfte Kontakte und Austausch zwischen den Teilnehmenden über die Tagungszeit bestehen blieben.

Die Vormittage der Tagung waren für die Präsentationen der 14 Teilnehmenden aus acht Ländern (Deutschland, Kanada, Niederlande, Österreich, Schweden, Schweiz, UK, USA) vorgesehen. An den Nachmittagen wurden in verschiedenen Workshops übergreifende Themen vertieft. Darin ging es vor allem um die Frage der Integration von Public Health Ethik in Forschung, Gesundheitspolitik und Lehre und darum, wie eine gute Vernetzung der wissenschaftlich Interessierten umgesetzt werden kann. Zu dem hohen fachlichen Niveau der Workshops trugen namhafte Gastdozierende bei: Angus Dawson (Birmingham), Peter Schröder-Bäck (Maastricht), Neema Sofaer (London) und Marcel Verweij (Utrecht). In einem öffentlichen Gastvortrag von Johannes van Delden (Utrecht) wurde die Frage nach einer Pflicht zur Grippeimpfung von Ärzten und Pflegenden diskutiert.

Dr. med. V. Wild $(\bowtie)$

Institut für Biomedizinische Ethik, Universität Zürich,

Pestalozzistr. 24, 8032 Zürich, Schweiz

E-Mail: wild@ethik.uzh.ch 
In den Vorträgen untersuchten die Teilnehmenden zum einen Public Health Methoden, Interventionen und Prinzipien aus ethischer Perspektive, zum anderen wurden die Methoden und Fundamente von Public Health Ethik diskutiert. Dabei wurden die verschiedensten Public Health Bereiche angesprochen, die ethisch relevante Spannungen mit sich bringen können, etwa die Impfung gegen H1N1 oder HPV (Geza Rothenberger, Deutschland), Online-Präventionsinstrumente gegen Gewalt an Kindern (Inge Lecluijze, Niederlande), Brustkrebs-Screening oder der Umgang mit dem Risiko von Phtalaten in Kinderspielzeug. Auch die Besteuerung von „ungesunden“ Nahrungsmitteln, Gesundheitsversorgung sozialer Randgruppen, Nichtraucherprogramme oder Adipositasprävention gaben Anlass zur ethischen Reflektion.

Public Health Interventionen betreffen meist die gesamte Bevölkerung oder bestimmte Bevölkerungsgruppen. Aus ethischer Perspektive ist daher demokratische Partizipation ein wünschenswertes Element, z. B. durch Bürgerforen. Viele Länder haben damit bisher wenig Erfahrungen, so auch Deutschland, und tatsächlich zeigen Studien, dass diese Methode ein kostenträchtiges und aufwändiges Unternehmen ist (Christopher McDougall, Kanada).

Um Public Health Interventionen umzusetzen, sind verschiedene Methoden in der Diskussion. Manche Interventionsprogramme wie etwa Nichtraucherkampagnen arbeiten damit, etwa durch Werbeclips, bei der Bevölkerung Stigma und Schuld auszulösen. Es ist jedoch fraglich, ob diese Methode je ethisch gerechtfertigt sein kann, da es dadurch wohl immer zur ungerechtfertigten Benachteiligung bestimmter Gruppen kommen kann (Kristin Voigt, UK). Eine weitere Methode, Public Health Interventionen umzusetzen, basiert auf dem Prinzip des libertären Paternalismus. Dabei sollen durch „,nudging“ (Anstupsen) Entscheidungen auf subtile Weise hin zu gesünderen Alternativen beeinflusst werden, ohne die Entscheidungsoptionen, also die persönliche Freiheit, einzuschränken. Allerdings ist für einen ideologiefreien und konstruktiven Diskurs über libertären Paternalismus begriffliche Genauigkeit und Klarheit über den normativen Gehalt notwendig (Kalle Grill, Schweden). Etliche weitere Prinzipien und Methoden von Public Health Interventionen sind aus ethischer Perspektive interessant. So müssen Methoden der Priorisierung bei Public Health Interventionen zum Beispiel bei Impfprogrammen auf ihre ethische Rechtfertigung hin untersucht werden (Jasper Littmann, UK). Bei potentiell risikobehafteten Innovationen ist das Vorsorgeprinzip ein gängiges Vorgehen, doch bedarf es bei seinem Einsatz einer sorgfältigeren moralischen Legitimation (Annette Rid, Schweiz). Etliche Public Health Interventionen und Leitlinien gehen von der Eigenverantwortung der Bevölkerung für ihre Gesundheit aus, doch ist auch dieses ein kontrovers diskutiertes Prinzip. Eine Möglichkeit ist es, ein ethisch angemessenes, nuanciertes Verständnis für eine Mit-verantwortung zu entwickeln (Harald Schmidt, USA). Zudem werden häufig in Epidemiologie, Public Health oder Evidenzbasierter Medizin (Martin Beyer, Deutschland) Kategorisierungen vorgenommen, die auf medizinisch-wissenschaftlichen Erkenntnissen beruhen. Jedoch sollte auch die ethische Dimension beachtet werden, zum Beispiel, ob mit einer Kategorisierung nicht soziale Ungerechtigkeit perpetuiert wird (Stephen John, UK).

Nicht nur die Methoden der Public Health Interventionen sind Gegenstand einer ethischen Diskussion, sondern auch die Methoden der Public Health Ethik selber. So werden für eine strukturierte ethische Analyse von Public Health Fragen methodische Rahmen diskutiert, die zum Beispiel beinhalten, welchen sozialen Wert die Intervention hat, wie gut umsetzbar sie ist oder ob deliberative und Fairness-Prozesse in die Umsetzung eingeflossen sind (Holly Taylor, USA). Zudem wird die Frage verhandelt, welches die ethischen Theorien im Hintergrund von Public Health und Public Health Interventionen sein sollen. Dabei wurde in den letzten Jahren die Bedeutung von Lebenskontext und sozialen Determinanten für Public Health zunehmend auch für die Theorie von Public Health Ethik wahrgenom- 
men. Der „capabilities approach“ von Martha Nussbaum ist eine Möglichkeit, Public Health auf ein ethisches Fundament zu stellen, das den Fokus weiter setzt als z. B. lediglich auf Maximierung von Gesamtnutzen (Martina Schmidhuber, Österreich). Theorien der sozialen Gerechtigkeit, die direkt auf Public Health Probleme zugeschnitten sind (z. B. [6]), können in Fragen zu Gesundheitsungleichheit plausible Argumente liefern (Verina Wild, Schweiz).

Angesichts der ethischen Dimension von Public Health ist Unterricht in Public Health Ethik notwendig, doch bisher gibt es wenig etablierte Curricula. Konzeptuelle Arbeit über die pädagogischen Mittel und zu vermittelnde philosophische Grundlagen ist notwendig (Alison Thompson, Kanada).

Insgesamt wurde während der Tagung deutlich, wie breit und interessant das Feld der Public Health Ethik angesichts der verschiedenen Anwendungsgebiete ist und wie ausbaufähig die ethischen Betrachtungen, auch im deutschen Kontext, noch sind. So zeigten die Diskussionen zu Konzepten, Methoden und Prinzipien vor allem eines: Es liegt noch viel Arbeit vor Public Health Ethik. Etablierte Prinzipien aus der Klinischen Ethik erscheinen aus der Bevölkerungsperspektive in einem neuen Licht: Autonomie, Paternalismus und Risiko-Nutzen-Bewertung müssen neu situiert werden. Aus einer Public Health Ethik Perspektive werden außerdem Prinzipien und Konzepte wie Solidarität, soziale Gerechtigkeit oder Reziprozität eine wesentlich stärkere Rolle spielen als bisher in der klinischen Ethik, doch müssen beispielsweise ihre moralische Rechtfertigung und ihr Verhältnis zur Freiheit und Verantwortung des Einzelnen noch besser bestimmt werden.

Durch die Publikation eines Sammelbandes im Springer International Verlag in der Reihe „Public Health Ethics“ werden die Ergebnisse 2012 auch einem breiteren Publikum zugänglich gemacht.

Über die fünf gemeinsamen Tage ist in intensiver Arbeitsatmosphäre und mit genügend Zeit zum informellen Austausch eine außerordentlich produktive und inspirierende Dynamik entstanden, die über das eigentliche Tagungsgeschehen hinaus anhält. Der Erfolg der Tagung ist der geschickt gewählten Thematik, der hervorragenden Organisation und der stimmigen Auswahl der Teilnehmenden durch Daniel Strech, Irene Hirschberg und Georg Marckmann geschuldet.

\section{Literatur}

1. Bayer R, Gostin LO, Jennings B, Steinbock B (Hrsg) (2007) Public health ethics: theory, policy and practice. Oxford University Press, Oxford

2. Buyx A, Huster S (Hrsg) (2010) Ethische Aspekte von Public Health. Ethik Med 22:175-299

3. Dawson A (Hrsg) (2011) Public health ethics. Key concepts and issues in policy and practice. Cambridge University Press, Cambridge

4. Gostin LO (2002) Public health law and ethics. A reader. University of California Press, New York

5. Holland S (2007) Public health ethics. Polity, Cambridge

6. Powers M, Faden RR (2006) Social justice. The moral foundations of public health and health policy. Oxford University Press, Oxford

7. Strech D, Marckmann G (Hrsg) (2010) Public Health Ethik. Lit, Berlin 